\title{
Secondary analysis of change in physical function after exercise intervention in older adults with hyperkyphosis and low physical function
}

Amy Gladin ${ }^{1}$, Wendy B. Katzman ${ }^{2 *}$, Yoshimi Fukuoka ${ }^{3}$, Neeta Parimi ${ }^{4}$, Shirley Wong ${ }^{2}$ and Nancy E. Lane ${ }^{5}$

\begin{abstract}
Background: Hyperkyphosis is common in older adults and associated with low physical function and reduced health related quality of life (HrQol). Improved kyphosis has been previously established in kyphosis-targeted interventions in randomized controlled trials in older adults with hyperkyphosis; however, evidence for improved physical function is conflicting. Few studies have investigated change in physical function after a targeted kyphosis intervention in older adults with low physical function. The primary aim in this descriptive study was to explore change in physical function after a progressive high-intensity 3-month targeted kyphosis exercise and posture training intervention in older adults with low physical function and hyperkyphosis. Secondary aims were to explore change in HrQol, spinal strength and spinal curvature, and adherence and safety of the intervention.

Methods: In this secondary analysis of the Specialized Center of Research (SCOR) Kyphosis randomized trial, 101 community dwelling older men and women with hyperkyphosis who completed the intervention were divided into a low function group (LFG) and high function group (HFG). Baseline characteristics were compared between LFG and HFG. Physical function, HrQol, spinal strength and spinal curvature (kyphosis and lordosis) pre/post intervention change scores were explored within and between groups. Adherence and adverse events were examined in the LFG and HFG.
\end{abstract}

Results: Twenty-six (26\%) older adults were LFG, mean Short Phyiscal Performance Battery (SPPB) 9.62 (SD = 1.17) points. At baseline, the LFG was older than HFG ( $p=0.005)$, experienced more pain, $(p=0.060)$, had worse physical function and $\mathrm{HrQol}(p \leq 0.001)$, and comparable kyphosis ( $p=0.640)$. SPPB changed 0.62 ( $95 \% \mathrm{Cl}:-0.20$ to 1.44) points in the LFG and - 0.04 (95\%Cl: -0.28 to 0.19 ) points in the HFG, $p=0.020$. Gait speed changed 0.04 (95\% Cl: -0.02 to 0.10$) \mathrm{m} / \mathrm{s}$ in the LFG. Kyphosis improved equally in both groups. Adherence to the intervention was $83 \%$ in the LFG and $79 \%$ in the HFG. There were no adverse events in either group.

(Continued on next page)

\footnotetext{
* Correspondence: Wendy.katzman@ucsf.edu

${ }^{2}$ Department of Physical Therapy and Rehabilitation Science, University of California, San Francisco, 1500 Owens Street, Suite 400, San Francisco, CA 94143, USA

Full list of author information is available at the end of the article
}

(C) The Author(s). 2021 Open Access This article is licensed under a Creative Commons Attribution 4.0 International License, which permits use, sharing, adaptation, distribution and reproduction in any medium or format, as long as you give appropriate credit to the original author(s) and the source, provide a link to the Creative Commons licence, and indicate if changes were made. The images or other third party material in this article are included in the article's Creative Commons licence, unless indicated otherwise in a credit line to the material. If material is not included in the article's Creative Commons licence and your intended use is not permitted by statutory regulation or exceeds the permitted use, you will need to obtain permission directly from the copyright holder. To view a copy of this licence, visit http://creativecommons.org/licenses/by/4.0/ The Creative Commons Public Domain Dedication waiver (http://creativecommons.org/publicdomain/zero/1.0/) applies to the data made available in this article, unless otherwise stated in a credit line to the data. 
(Continued from previous page)

Conclusions: Older adults with low physical function and hyperkyphosis may improve physical function after a kyphosis targeted intervention. Older adults with low physical function may safely participate in targeted highintensity kyphosis exercise and posture training. This observation needs to be confirmed in larger adequately powered studies.

Trial registration: Clinicaltrials.gov identifier: NCT01766674.

Keywords: Hyperkyphosis, Physical function, Older adults, Health related quality of life

\section{Introduction}

Age-related hyperkyphosis, commonly defined by a thoracic spine Cobb angle curvature of 40 degrees or greater, progresses with age and affects up to $40 \%$ of older adults [1]. Hyperkyphosis in older adults has been associated with impaired physical function, reduced health-related quality of life (HrQol), increased falls and fracture risk and is a predictor of all-cause mortality in women [110]. Numerous cross-sectional and longitudinal studies have demonstrated hyperkyphosis is associated with slowed gait speed cross-sectionally and is predictive of worsening chair-stand time and Timed Up and Go (time to rise from chair, walk $10-\mathrm{m}$ turn and return to sit) performance longitudinally in adjusted models [2-6, 11]. Furthermore, hyperkyphosis has been identified as a 'new' geriatric syndrome, thus targeting hyperkyphosis as an impairment may contribute to slowing the progression to physical frailty [12].

It is theorized that hyperkyphosis causes an anterior displacement of the center of gravity, which affects physical function characteristics and balance, and in turn negatively impacts physical function [13]. Therefore, it has been hypothesized that interventions that reduce kyphosis may also improve physical function; however, few randomized controlled trials targeting improvement of kyphosis have reported a significant increase in physical function despite successfully reducing kyphosis [14-18]. Previous trial results could be explained by a ceiling effect in high functioning cohorts of individuals who were already functioning above age-matched normative values for physical function. Further investigation of the effects of a targeted kyphosis intervention on physical function in older adults with low function and hyperkyphosis may inform future treatment for older adults at risk for physical function decline and frailty.

Since it is unknown if older adults with hyperkyphosis and low physical function who undergo a targeted kyphosis intervention will also improve physical function, we performed a secondary analysis using data from the Specialized Center of Research (SCOR) randomized controlled trial that investigated change in kyphosis, physical function and HrQoL in older adults with hyperkyphosis after a 3-month targeted high-intensity kyphosis exercise and posture training intervention [14]. We categorized participants post-hoc into low and high physical function groups according to baseline physical function scores. We hypothesized that older adults with hyperkyphosis, low physical function, and who are transitioning to frailty would improve physical function after a targeted kyphosis intervention. Our primary aim was to explore change in physical function in older adults with hyperkyphosis and low physical function, and whether they responded differently than adults with higher function. We also explored change in HrQol, spinal strength and spinal curvature in low versus high function groups. Lastly, we explored the feasibility of conducting a targeted high-intensity kyphosis intervention in older adults with hyperkyphosis and low physical function by comparing adherence to the intervention and safety in both low and high function groups. We used change in physical function scores in the low function group to determine a sample size needed to test the hypothesis in a future, fully powered trial.

\section{Methods \\ Study design}

This secondary data analysis included participants $(n=$ 101) who completed the SCOR kyphosis intervention in a randomized controlled waitlist design trial [14]. In the SCOR trial, between group comparisons were made between the active and control group at 3-months, then the waitlist group received the intervention and a 6month assessment to investigate within subject change after receiving the intervention for all participants. For the secondary analysis, we calculated a baseline Short Physical Performance Battery (SPPB) score post-hoc using subcomponents of baseline measurements to divide the SCOR cohort into a low functioning group (LFG) and a high functioning group (HFG). The SPPB is a lower extremity strength, mobility and balance physical performance measure, and a composite measure of gait speed, Five-times Sit to Stand and ability to stand with feet together, feet in half tandem and feet in full tandem for $10 \mathrm{~s}$ for each condition [19]. Each component is scored on an ordinal scale from 0 to 4 , where 0 represents lowest ability and 4 represents highest ability, with a maximum score of 12 possible points. The SPPB is predictive of future mobility decline and incident 
activities of daily living disability [19-21]. The definition of low physical function was operationalized by an established cut-off score of 10 or less on the SPPB that identifies older adults who are at-risk for mobility decline [20, 22]. A score of 10 or less has been identified as the best cut-off point for the determination of the physical frailty process with a likelihood radio of 1.59 [20, 22]. An SPPB score of 11 or 12 was categorized as the HFG. The SPPB has high levels of reliability, good to moderate concurrent validity with quality of life, strength, muscle power and mobility and scores less than 10 are predictive of all-cause mortality [23, 24].

\section{Study participants}

Participants in the original SCOR trial $(n=112)$ were recruited from a university-based medical center and an integrated managed-care center in San Francisco from January 2013 to June 2015 and included communitydwelling adults age 60 or greater with hyperkyphosis > 40 degrees, English language proficient, able to walk 1 block without an assistive device and rise from a chair without their hands [14]. Participants were excluded from the SCOR trial if they were unable to actively extend their thoracic spine by at least 5 degrees or had cognitive impairment [25]. Participants were initially randomized to an active $(n=57)$ or waitlist control $(n=$ 55) group; however, 9 withdrew within the first week due to lack of time or interest and 2 did not have analyzable baseline radiographs for Cobb angle measurements. Participants $(n=101)$ who completed the trial were included in the secondary analysis. The trial protocol was approved by the Institutional Review Boards at the University of California, San Francisco and Kaiser Permanente Northern California. Written informed consent was obtained from all participants. The study protocol and methods were performed in accordance with the guidelines and regulations of the Declaration of Helsinki.

\section{Intervention groups}

The SCOR active intervention was a physical therapist led group targeted kyphosis exercise and posture training program for 1-h twice weekly for 3 months [14]. A licensed physical therapist led the intervention and provided participants with verbal feedback and a trained research assistant provided additional supervision and feedback. A ratio of 5 study participants to 1 staff member was maintained during every intervention session. The active intervention included progressive highintensity spinal and lower extremity strengthening exercise, thoracic spine and lower extremity range of motion exercise, and posture training. Participants were asked to practice good posture during activities of daily living outside the intervention and provided with an educational handout with pictures to reinforce good posture during activities of daily living. Details on the exercise and posture training intervention have been previously published [26]. A wait-list control group received usual care during the initial 3-months and received the active intervention after the 3-month waitlist period, thus all participants received the intervention.

\section{Demographic and other measures}

Prior to randomization in the original SCOR trial, participants provided demographic and health information via self-report (age, sex, education, co-morbidities). Height and weight were collected using standard measures, and body mass index was calculated. Bone mineral density of the hip and spine was measured using dual-energy X-ray absorptiometry (GE Lunar Prodigy, Madison, WI, USA). Baseline standing lateral spine radiographs were were performed at a University radiology clinic and followed a standardized protocol for measurement of standing lateral spine [27]. Participants were evaluated for prevalent vertebral fractures and diffuse idiopathic skeletal hyperostosis (DISH) by experienced assessors [28-30].

\section{Outcome measures}

SCOR study outcome measures were performed at baseline, 3-month and 6-month timepoints (for the waitlist group only). The waitlist group received the intervention after the 3-month measurement. All measurements were performed by trained examiners masked to group allocation (no waitlist, waitlist).

\section{Physical function}

Performance-based physical function was assessed using the modified Physical Performance Test, 4-m walk test, Timed Up and Go (TUG) and 6 min Walk (6 MW) tests [31-33]. The modified Physical Performance Test included 7 standardized timed tasks: 50-ft floor walk, donning and doffing a lab coat, picking up penny from floor, Five-times Sit to Stand test from a $41 \mathrm{~cm}$ chair without using upper extremities, lifting a 7-pound book, climbing one flight of stairs, standing balance and two untimed tasks: climb up/down 4 flights of stairs and performing a 360 degree turn [33]. Each component is scored 0 to 4 with a maximum score of 36 . Scores 25 to 31 indicate mild frailty [34]. The 4-m walk test (gait-speed) measures time to walk $4 \mathrm{~m}$ (meter/second) [31]. The $6 \mathrm{MW}$ test measures distance (meters) walked in $6 \mathrm{~min}$ [31]. The TUG measures time(s) to rise from a chair, walk 10-m turn and return seated to the chair [31]. The fourmeter walk test, $6 \mathrm{MW}$ and TUG are well described in the literature and have good to excellent reliability among older adults with arthritis [31, 32]. The PatientReported Outcome Measuremement Information System (PROMIS) Physical Function questionnaire is scored using a t-score metric and is calibrated to have the population mean be a $\mathrm{t}$-score of 50 with the standard 
deviation set to be 10 [35]. Scores range from 0 to 100 and higher $\mathrm{t}$-scores indicate improved physical function. The SPPB score was calculated post-hoc from subcomponents of the modified Physical Performance Test and 4-m walk test data.

\section{Health Related Quality of Life (HrQol)}

Participants completed a battery of patient reported HRQoL outcomes including, PROMIS Global Health Scale v.1.0 (both physical and mental health individual scores), the modified Scoliosis Research Society 30, selfimage domain and the Physical Activity Scale for the Elderly (PASE) [36-38]. For all HrQol measures, an increase in score indicates an improvement within the specific domain (see Table 2 for score ranges). Pain and self-rated general health outcomes were extracted from single questions within the PROMIS Scale v.1.0 - Global Health [39]. The pain measurement within the PROMIS Global Health questionnaire utilizes a visual analogue scale and states 'In the past 7 days, how would you rate your pain on average' 0 indicates 'no pain' and 10 indicates 'worst imaginable pain'.

\section{Spinal strength}

Spinal strength was measured using the Biodex 3 (Biodex Medical Systems Inc., Shirley, NY, USA), a computerized dynamometer for spinal flexors and spinal extensors [40]. Spinal endurance was measured with the Timed Loaded Standing test, a combined measure of trunk and arm endurance, and is quantified as the time in seconds able to hold a 2-pound dumbbell in each hand with both shoulders flexed to 90 degrees and elbows extended to neutral [41].

\section{Spinal curvature and cobb angle kyphosis}

Clinical measures of thoracic kyphosis and lumbar lordosis were measured in a usual standing posture with the Debrunner Kyphometer (Techmedica Inc., Camarillo, CA, USA) [42, 43]. The Debrunner Kyphometer measures an external Cobb angle with the feet of the kyphometer placed over the T2/3 and T11/12 interspaces (Fig. 1). Cobb angle of kyphosis measurements were made from standing lateral spine radiographs according to standardized protocols by an experienced radiologist [27].

\section{Adherence and safety}

Adherence to the intervention sessions, adverse events and non-reportable events were monitored by the study coordinator, who administered a standardized questionnaire on a weekly basis during the active intervention. Mean adherence to sessions was calculated as a percentage based upon the number of sessions attended out of the 24 sessions possible. Adverse events are defined as any unfavorable medical occurrence and problems that

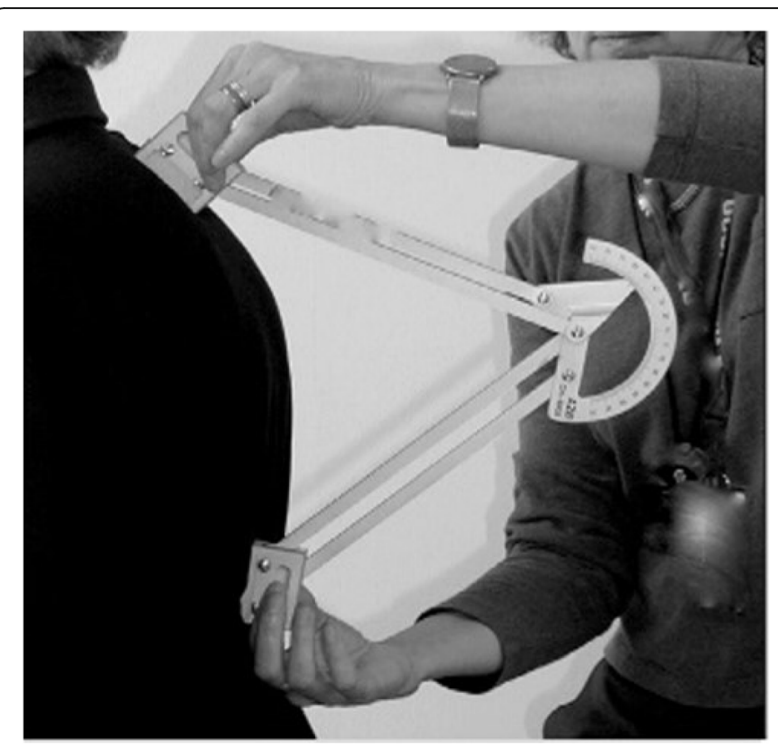

Fig. 1 Debrunner Kyphometer. The Debrunner Kyphometer measures an external Cobb angle with the feet of the kyphometer placed over the T2/3 and T11/12 interspaces

are possibly related to study participation serious or unexpected. Non-reportable events are expected symptoms that may occur during the intervention and described in the study protocol and disclosed in the consent. Examples of nonreportable events in this study are muscle or joint soreness or exacerbation of previous injuries associated with the intervention and resolved within an expected duration of time [44].

\section{Data analysis for secondary analysis}

Baseline characteristics were compared between the LFG and HFG using t-tests or Wilcoxon nonparametric tests for continuous variables and the $\chi^{2}$ statistic for categorical variables. We calculated mean differences and 95\% confidence intervals for change pre/post treatment unadjusted for any covariates for all outcome measures in the LFG and HFG. We compared the pre/post intervention change scores in the LFG and HFG using t-tests. In exploratory analysis we performed a sensitivity analysis and adjusted for age and gender. $P$-values $<0.05$ were considered statistically significant. Analyses were conducted using SAS Version 9.4 (SAS Inc., Cary, NC). In this post-hoc secondary analysis, we did not have power to detect significant differences within the groups based upon the small sample size. We determined sample sizes needed for a future study to test the hypothesis that older adults with hyperkyphosis and low physical function will improve physical function on the SPPB after a kyphosis exercise and posture training intervention. Sample size calculations were conducted using PASS 15.0.3 software (Power Analysis \& Sample Size, NCSS Software, Kaysville, UT, USA). 


\section{Results}

There were more men in the LFG versus HFG, $54 \%$ vs $36 \%, p=0.110$. The LFG was older than the HFG, 72.4 $(\mathrm{SD}=6.5)$ years versus $68.8(\mathrm{SD}=5.15)$ years, $p=0.005$, and reported poorer general health, $p=0.006$, and more pain $3.3(\mathrm{SD}=2.7)$ points in the HFG and $2.0(\mathrm{SD}=1.7)$ in the LFG, $p=0.060$, (Table 1).

Twenty-six percent $(n=26)$ adults had low function, mean SPPB score $9.62(\mathrm{SD}=1.2)$ points, and $74 \%(n=$ $75)$ were considered high functioning, mean SPPB score $11.37(\mathrm{SD}=0.7$ ) points (Table 2). At baseline, all physical function and HrQol scores were lower in the LFG versus the HGF, $p=0.017$, except physical activity was similar in both groups, $p=0.330$. Trunk endurance was worse in the LFG, $p=0.017$. There were no differences in baseline levels of kyphosis, $p=0.216$.

\section{Within group change in physical function in low function} group

There were no significant within-group changes in SPPB, 4-m gait speed, modified Physical Performance Test, TUG, $6 \mathrm{MW}$ or PROMIS physical function in the LFG in the unadjusted analysis (Table 3). The mean SPPB score changes for the LFG was $0.62(95 \% \mathrm{CI}$ : -0.2 to 1.44) points and 4-m gait speed mean change was 0.04 (95\%CI: -0.02 to 0.10$) \mathrm{m} / \mathrm{s}$. TUG mean change was 0.2 (95\%CI: -0.6 to 0.3 ) seconds and the $6 \mathrm{MW}$ mean change was 1.8 (95\%CI: -14.0 to 17.6$)$ meters. After controlling for age, the LFG (mean SPPB $=9.6$ ) improved 0.77 (95\%CI: 0.23 to 1.3 ) points (Additional file 1). After controlling for age and sex, improvement in SPPB increased to $0.84(95 \% \mathrm{CI}: 0.31$ to 1.38$)$ points (Additional file 2). After controlling for age and age and sex, there were no significant within-group changes in the LFG in 4-m gait speed, modified Physical Performance Test, TUG, $6 \mathrm{MW}$ or PROMIS physical function.

\section{Within group change in HrQol, spinal strength and spinal curvature}

Self-image, a measure of HrQol, improved within both groups 0.19 (95\%CI: 0.02 to 0.4 ) points in the LFG and 0.24 (95\%CI: 0.1 to 0.3 ) points in the HFG (Table 3). Spinal extensor strength had a mean change score of 6.8 (95\%CI: -16.12 to 2.56$)$ percent in LFG and

Table 1 Study participant characteristics at baseline in the HFG and LFG

\begin{tabular}{|c|c|c|c|c|c|}
\hline \multirow[t]{2}{*}{ Variable } & \multirow[t]{2}{*}{ Category } & \multirow{2}{*}{$\begin{array}{l}\text { Low Function Group } \\
\text { (LFG) } \\
n=26 \\
N(\%) \text {, mean } \pm \text { SD }\end{array}$} & \multirow{2}{*}{$\begin{array}{l}\text { High Function Group } \\
\text { (HFG) } \\
n=75 \\
\mathrm{~N}(\%) \text {, mean } \pm \text { SD }\end{array}$} & \multirow{2}{*}{$\begin{array}{l}\text { LFG vs. } \\
\text { HFG* } \\
p \text {-value }\end{array}$} & \multirow{2}{*}{$\begin{array}{l}\text { Entire Cohort } \\
n=101 \\
\mathrm{~N}(\%), \\
\text { mean } \pm \mathrm{SD}\end{array}$} \\
\hline & & & & & \\
\hline Age (years) & & $72.4 \pm 6.6$ & $68.8 \pm 5.2$ & 0.005 & $69.7 \pm 5.7$ \\
\hline Gender & Female & $12(46)$ & $48(64)$ & 0.110 & $60(59)$ \\
\hline \multirow[t]{3}{*}{ Vertebral Fracture } & none & $22(85)$ & $65(87)$ & 0.741 & $87(86)$ \\
\hline & 1 & $2(8)$ & $7(9)$ & & $9(9)$ \\
\hline & 2 & $2(8)$ & $3(4)$ & & $5(4.95)$ \\
\hline $\begin{array}{l}\text { Diffuse idiopathic hyperostosis (DISH) present } \\
\text { (yes) }\end{array}$ & & $7(28)$ & $15(21)$ & 0.461 & $22(23)$ \\
\hline Body Mass Index(kilograms/meter²) & & $27.7 \pm 3.9$ & $25.7 \pm 4.1$ & 0.037 & $26.2 \pm 4.1$ \\
\hline Bone mineral density total hip t-score & & $-0.5 \pm 1.3$ & $-0.9 \pm 1$ & 0.074 & $-0.85 \pm 1.1$ \\
\hline Bone mineral density total spine t-score & & $0.7 \pm 2.8$ & $-0.5 \pm 1.8$ & 0.064 & $-0.2 \pm 2.2$ \\
\hline Race & Caucasian & $25(96)$ & $69(92)$ & 0.472 & $94(93)$ \\
\hline \multirow[t]{2}{*}{ Education } & $\begin{array}{l}\text { High school, some } \\
\text { College }\end{array}$ & $2(8)$ & $11(15)$ & 0.360 & $13(13)$ \\
\hline & $\begin{array}{l}\text { College, professional } \\
\text { degree }\end{array}$ & $24(92)$ & $64(85)$ & 0.360 & $88(87)$ \\
\hline $\begin{array}{l}\text { Pain Score from PROMIS scale 1.0-Global health, } \\
0-10 \text { (points) }\end{array}$ & & $3.3 \pm 2.7$ & $2 \pm 1.7$ & 0.060 & $2.3 \pm 2.1$ \\
\hline \multirow{4}{*}{$\begin{array}{l}\text { Self-rated health from PROMIS scale 1.0-Global } \\
\text { Health }\end{array}$} & Fair & $5(19)$ & $3(4)$ & 0.006 & $8(9)$ \\
\hline & Good & $12(46)$ & $19(25)$ & & $31(31)$ \\
\hline & Very Good & $7(27)$ & $41(55)$ & & $48(48)$ \\
\hline & Excellent & $2(8)$ & $12(16)$ & & $14(14)$ \\
\hline Co-morbidities & 2 or more & $10(38)$ & $30(40)$ & 0.890 & $40(40)$ \\
\hline
\end{tabular}


Table 2 Means of measures at baseline stratified by HFG, LFG and entire cohort

\begin{tabular}{|c|c|c|c|c|}
\hline \multirow[t]{2}{*}{ Outcome measures } & $\begin{array}{l}\text { Low Function Group } \\
\text { (LFG) } \\
n=26\end{array}$ & $\begin{array}{l}\text { High Function Group } \\
\text { (HFG) } \\
n=75\end{array}$ & $\begin{array}{l}\text { LFG vs. } \\
\text { HFG* }\end{array}$ & $\begin{array}{l}\text { Entire } \\
\text { cohort } \\
n=101\end{array}$ \\
\hline & Mean $( \pm S D)$ & Mean $( \pm S D)$ & $P$-value & Mean $( \pm S D)$ \\
\hline \multicolumn{5}{|l|}{ Physical Function } \\
\hline Short Physical Performance Battery (SPPB) (0-12 points) & $9.62(1.2)$ & $11.37(0.7)$ & $<0.001$ & $10.92(1.1)$ \\
\hline 4-m gait speed (meters/second) & $1.14(0.2)$ & $1.38(0.34)$ & $<0.001$ & $1.32(0.3)$ \\
\hline Modified PPT (0-36 points) & $31.4(2.7)$ & $33.8(1.7)$ & $<0.001$ & $33.2(2.2)$ \\
\hline Timed Up and Go (seconds) & $8.4(1.6)$ & $7.1(1.5)$ & $<0.001$ & $7.4(1.7)$ \\
\hline Six Minute Walk Test (meters) & $471.7(77.2)$ & $524.9(95.9)$ & 0.008 & $511.6(94.1)$ \\
\hline PROMIS Physical function t-score (0-100) & $44.8(4.7)$ & $50.1(7.6)$ & $<0.001$ & $48.7(7.3)$ \\
\hline \multicolumn{5}{|l|}{ Health-related Quality of Life } \\
\hline SRS 30 Self-image (0-5 points) & $3.33(0.49)$ & $3.69(0.56)$ & 0.016 & $3.53(0.55)$ \\
\hline PROMIS Global Health Scale (0-100) & $35.8(6.5)$ & $40.7(4.7)$ & 0.001 & $39.5(5.6)$ \\
\hline PROMIS Global Health Scale, Mental Health t-score (0-100) & $48.8(8.1)$ & $54.1(7.5)$ & 0.003 & $52.8(7.9)$ \\
\hline PROMIS Global Health Scale, Physical Health t-score (0-100) & $47.9(7.5)$ & $53.6(5.6)$ & $<0.001$ & $52.2(6.6)$ \\
\hline PASE activity level (0-793) points & $102.1(60.5)$ & $111.0(53.2)$ & 0.330 & $108.7(54.9)$ \\
\hline $\begin{array}{l}\text { Pain Score 0-10 from PROMIS Global Health scale (0-10 } \\
\text { points) }\end{array}$ & $3.3(2.7)$ & $2.0(1.68)$ & 0.060 & $3.4(2.1)$ \\
\hline \multicolumn{5}{|l|}{ Spinal Strength } \\
\hline Spinal flexion strength (percent peak torque/body weight) & $28.8(11.4)$ & $32.7(11.3)$ & 0.119 & $31.7(11.4)$ \\
\hline $\begin{array}{l}\text { Spinal extensor strength (percent peak torque/body } \\
\text { weight) }\end{array}$ & $64.2(16.2)$ & $71.0(22.0)$ & 0.238 & $69.2(20.8)$ \\
\hline Timed Loaded Standing (seconds) & $112.0(49.9)$ & $138.4(49.0)$ & 0.017 & $131.0(50.3)$ \\
\hline \multicolumn{5}{|l|}{ Spinal Curvature } \\
\hline Cobb angle of kyphosis (degrees) & $56.8(13.3)$ & $55.5(11.7)$ & 0.640 & $55.9(12.2)$ \\
\hline Kyphosis derived from kyphometer (degrees) & $53.6(6.2)$ & $51.5(7.8)$ & 0.216 & $52.0(7.4)$ \\
\hline Lordosis derived from kyphometer (degrees) & $25.9(12.2)$ & $31.7(11.4)$ & 0.028 & $30.2(11.8)$ \\
\hline
\end{tabular}

SD Standard Deviation, Modified PPT Physical Performance Test, SRS Scoliosis Research Society, PASE Physical Activity Scale for the Elderly, PROMIS PatientReported Outcome Measurement Information System, HFG high functioning group, LFG low functioning group, group * $p$ values for comparison between LFG and HFG

improved 6.0 (95\%CI: 1.0 to 11.0 ) percent in the HFG. Spinal endurance measured by the Timed Loaded Standing mean change was -8.8 (95\% CI: -23.3 to 5.7 ) seconds in the LFG and 5.2 (95\%CI: -1.6 to 12$)$ seconds in the HFG. Kyphometer measured kyphosis improved 3.1 (95\%CI: -5.2 to -0.9$)$ degrees and -3.7 (95\%CI: 5.0 to -2.4 ) degrees in the LFG and HFG respectively. PROMIS Global health, physical health scale improved 2.3 (95\%CI: 0.25 to 4.39 ) points in the age and sex adjusted model in the LFG and 2.2 (95\%CI: 0.15 to 4.21 ) points in the age adjusted model in the LFG (Additional files 1 and 2).

\section{Between group change in physical function in low function versus high function}

Comparing change pre/post intervention in the LFG and HFG, the LFG improved SPPB more than the HFG, $p=$ 0.020 (Table 3). The mean SPPB score changes for the LFG was 0.62 (95\%CI: -0.2 to 1.44$)$ points and -0.04
(95\%CI: -0.02 to 0.10$) \mathrm{m} / \mathrm{s}$ in the $\mathrm{LFG}$ and $-0.01(95 \% \mathrm{CI}$ : -0.06 to 0.04$) \mathrm{m} / \mathrm{s}$ in the HFG, $p=0.406)$, TUG, $(0.2$ (95\%CI: -0.6 to 0.3 ) seconds in the LFG and 0.04 (95\%CI: -0.2 to 0.3 ) seconds in the HFG, $p=0.370$ ), or 6MW, (1.8 (95\% CI: -14.0 to 17.6$)$ meters in the LFG and 8.8 (95\%CI: -4.6 to 22.1 ) meters in the HFG, $p=0.265$ ) in the unadjusted analysis (Table 3). Comparing change pre/post intervention in the LFG and HFG, the LFG improved SPPB more than the HFG in the ageadjusted model, $p=0.008$ and the age and sex adjusted model, $p=0.003$ (Additional files 1 and 2). After controlling for age, the LFG (mean SPPB $=9.6$ ) improved 0.77 (95\% CI: 0.23 to 1.3 ) points while the HFG (mean $\mathrm{SPPB}=11.4$ points) changed - 0.093 (95\% CI: -0.04 to 0.22 ) points (Additional file 1). After controlling for age and sex, improvement in SPPB increased further to 0.84 (95\%CI: 0.31 to 1.38 ) points and changed -0.12 (95\%CI: -0.43 to 0.19 ) points in the HFG (Additional file 2). 
Table 3 Change scores pre/post intervention in outcome measures in the HFG, LFG and overall cohort with confidence intervals (Cl)

\begin{tabular}{|c|c|c|c|c|}
\hline & $\begin{array}{l}\text { Low -Function group } \\
\text { (LFG) }\end{array}$ & $\begin{array}{l}\text { High-Function group } \\
\text { (HFG) }\end{array}$ & $\begin{array}{l}\text { LFG vs } \\
\text { HFG* }\end{array}$ & Entire cohort \\
\hline & $\begin{array}{l}\text { Mean difference }(95 \% \\
\mathrm{Cl})\end{array}$ & $\begin{array}{l}\text { Mean difference }(95 \% \\
\mathrm{Cl})\end{array}$ & $p$-value & Mean $(95 \% \mathrm{Cl})$ \\
\hline \multicolumn{5}{|l|}{ Physical Function } \\
\hline Short Physical Performance Battery (SPPB) (0-12 points) & $0.62^{\mathrm{a}}(-0.20$ to 1.44$)$ & $-0.04(-0.28$ to 0.19$)$ & 0.020 & $\begin{array}{l}0.13(-0.14 \text { to } \\
0.40)\end{array}$ \\
\hline 4-m gait speed (m/s) & $0.04^{\mathrm{a}}(-0.02$ to 0.1$)$ & $-0.01(-0.06$ to 0.04$)$ & 0.406 & $0(-0.04$ to 0.04$)$ \\
\hline Modified PPT (0-36 points) & $-0.29(-2.7$ to 2.1$)$ & $-1.3(-3.0$ to 0.3$)$ & 0.166 & $-1.1(-2.4$ to 0.3$)$ \\
\hline Time up and go (seconds) & $-0.2(-0.6$ to 0.3$)$ & $0.04(-0.2$ to 0.3$)$ & 0.370 & $-0.01(-0.2$ to 02$)$ \\
\hline 6 Minute walking test (meters) & $1.8(-14.0$ to 17.6$)$ & $8.8(-4.6$ to 22.1$)$ & 0.265 & $7.0(-3.6$ to 17.6$)$ \\
\hline PROMIS Physical Function t-score (0-100 points) & $0.91(-5.5$ to 2.3$)$ & $2.0(0.5$ to 3.5$)$ & 0.353 & 1.7 (0.5 to 2.9$)$ \\
\hline \multicolumn{5}{|l|}{ Health-related Quality of Life } \\
\hline SRS 30 Self-image (0-5 pts) & 0.19 (0.02 to 0.4$)$ & 0.24 (0.1 to 0.3$)$ & 0.473 & 0.23 (0.1 to 0.3$)$ \\
\hline PROMIS Global Health Scale (0-100 points) & $1.0(-0.8$ to 2.8$)$ & $0.3(-0.5$ to 1.1$)$ & 0.820 & $0.5(-0.2$ to 1.2$)$ \\
\hline $\begin{array}{l}\text { PROMIS Global Health scale, Mental Health t-score (0-100 } \\
\text { points) }\end{array}$ & $1.19(-0.8$ to 3.2$)$ & $0.3(-0.9$ to 1.5$)$ & 0.536 & $0.52(-0.5$ to 1.6$)$ \\
\hline $\begin{array}{l}\text { PROMIS Global Health scale, Physical Health t-score (0- } \\
100 \text { points) }\end{array}$ & $1.8(-0.3$ to 3.9$)$ & $0.6(-0.5$ to 1.8$)$ & 0.194 & $0.9(-0.06$ to 1.9$)$ \\
\hline PASE activity (0-793) points & $6.3(-14.0$ to 26.6$)$ & $1.7(-8.2$ to 11.6$)$ & 0.928 & $2.9(-5.9$ to 11.7$)$ \\
\hline $\begin{array}{l}\text { Pain Score 0-10 from PROMIS Global Health scale (0-10 } \\
\text { points) }\end{array}$ & $-0.2(-0.9$ to 0.5$)$ & $-0.3(-0.6$ to 0.06$)$ & 0.54 & $\begin{array}{l}-0.26(-0.5 \text { to } \\
0.03)\end{array}$ \\
\hline \multicolumn{5}{|l|}{ Spinal Strength } \\
\hline Spinal Flexion (percent peak torque/bodyweight) & $-0.09(-3.4$ to 3.2$)$ & $1.5(-0.1$ to 3.2$)$ & 0.515 & $1.12(-0.4$ to 2.6$)$ \\
\hline Spinal extensor (percent peak torque/bodyweight) & $-6.8(-16.1$ to 2.6$)$ & $6.0(1.0$ to 11.0$)$ & 0.018 & $2.7(-1.8$ to 7.2$)$ \\
\hline Time loaded standing (seconds) & $-8.8(-23.3$ to 5.7$)$ & $5.2(-1.6$ to 12$)$ & 0.080 & $1.6(-4.7$ to 7.8$)$ \\
\hline \multicolumn{5}{|l|}{ Spinal Curvature } \\
\hline Cobb Angle (degrees) & $-0.6(-2.0$ to 0.9$)$ & $-1.6(-2.7$ to -0.4$)$ & 0.390 & $\begin{array}{l}-1.3(-2.2 \text { to }- \\
0.4)\end{array}$ \\
\hline Kyphosis (degrees) & $-3.1^{\mathrm{a}}(-5.2$ to -0.9$)$ & $-3.7^{\mathrm{a}}(-5.0$ to -2.4$)$ & 0.409 & $\begin{array}{l}-3.5(-4.6 \text { to }- \\
2.4)\end{array}$ \\
\hline Lordosis (degrees) & $0.4(-2.0$ to 2.9$)$ & $-1.5(-3.0$ to 0.03$)$ & 0.229 & $-1.0(-2.3$ to 0.3$)$ \\
\hline
\end{tabular}

Cl confidence interval, Modified PPT Physical Performance Test, SRS Scoliosis Research Society, PASE Physical Activity Scale for the Elderly, HFG high functioning group, LFG low functioning group

a denotes change scores surpassing minimum clinical change estimates (SPPB .03 to .08 points and gait speed .03 to $.06 \mathrm{~m} / \mathrm{s}$ and kyphosis Minimum Detectable Change 2.51 degrees) $[18,45,46]$

${ }^{*} p$ values for comparison between LFG and HFG

\section{Between group change in HrQol, spinal strength and spinal curvature}

Comparing change pre/post intervention in the LFG and HFG, there no significant between-group changes in selfimage, $p=0.473$ (Table 3). There were significant betweengroup changes in spinal extensor strength, $p=0.018$. There were no significant between-group changes in Timed Loaded standing, $p=0.800$. Both groups improved equally in kyphometer measured kyphosis, $p=0.409$. There was significant between-group changes in spinal extensor strength in the age-adjusted model, $p=0.036$ and borderline between-group change in the age and sex adjusted models, $p=0.050$.

\section{Feasibility}

Adherence during the 3 month intervention was $83 \%$ in the LFG and $79 \%$ in the HFG (Table 4). There were no adverse events in either group. 69\% (18/26) of the low function participants and 68\% (51/76) of the high function participants had non-reportable events including including pain and stiffness several hours to days after exercise, often from a pre-existing musculoskeletal complaint, which resolved within an expected period of time [44]. The LFG reported an average 2.83 events per person and the HFG reported 2.35 events per person. 
Table 4 Adherence and safety/adverse events in the LFG and HFG

\begin{tabular}{lll}
\hline & \multicolumn{1}{c}{ Low-Function group (LFG) } & $\begin{array}{l}\text { High-Function group (HFG) } \\
\mathbf{N}=\mathbf{7 5}\end{array}$ \\
\hline Mean adherence to sessions (percent) & $\mathbf{N = 2 6}(83)$ & $19(79)$ \\
Number of people reporting non-reportable events ${ }^{\mathrm{a}}$ (percent) & $18(69)$ & $51(68)$ \\
Number non-reportable events & 51 & 120 \\
Range of non-reportable events per person & 1 to 8 & 1 to 8 \\
Mean number of non-reportable events per person & 2.8 & 2.4 \\
Number of people reporting adverse events & 0 & 0
\end{tabular}

LFG low functioning group, HFG high functioning group

${ }^{a}$ Non-reportable events are symptoms that may occur during the intervention and disclosed in the consent, and resolve within an expected duration of time

${ }^{\mathrm{b}}$ Adverse events are defined as any untoward or unfavorable medical occurrence

\section{Sample size calculation}

Based on a 2-by-2 repeated measures design a sample size of $n=138$ (69 in invervention, 69 in control group) achieves $80 \%$ power to detect a difference in mean change of 0.6 points on the SPPB at a 0.050 significance level (alpha) using a two-sided, two-sample t-test (Additional file 3).

\section{Discussion}

The primary purpose of this secondary data analysis was to explore the hypothesis that targeting kyphosis will improve physical function in a low functioning cohort of older adults with hyperkyphosis who are transitioning to physical frailty. While there were no statistically significant changes in physical function in the participants with low physical function in the unadjusted analysis, there were significant improvements in SPPB in the adjusted models, and there were clinically significant improvements for the mean SPPB score and gait speed after the targeted kyphosis intervention in the LFG in both unadjusted and adjusted models. There were small improvements in self-image in both groups. Kyphosis improved across both LFG and HFG, even though spinal extensor strength and Time Loaded Standing had relative, negative changes the LFG and relative positive changes in the HFG. Adherence to the intervention, adverse events and non-reportable events were similar in both groups suggesting the intervention is acceptable and safe in a low functioning cohort.

As we hypothesized, SPPB improved a clinically significant amount in the LFG. SPPB changed 0.62 (95\%CI: -0.2 to 1.44$)$ points in the unadjusted model and improved 0.77 (95\%CI: 0.23 to 1.3 ) and 0.84 (95\%CI: 0.31 to 1.38) points in the age and age and sex adjusted models in the LFG. This is similar to 2 previous randomized studies that included lower functioning adults $[17,18]$. Benedetti et al. [17] performed a targeted exercise program among older adults with hyperkyphosis, and both active and control groups improved SPPB while only the control group improvement was significant $(p=0.03)$. Baseline score for SPPB in the active group was $10.33(\mathrm{SD}=1.17)$ points and improved 0.74 points after the intervention, and the control group baseline score was $9.38(\mathrm{SD}=1.04)$ points and improved 1.08 points, consistent with the $0.62(\mathrm{SD}=2.00)$ point change in our LFG. However, both groups in the Benedetti trial received an exercise program. The active group received targeted spinal strengthening while the control group received a global posture training program, which may explain why both groups improved. Jang et al. [18] tested the efficacy of an 8-week kyphosis correction exercise program in a frailer group than our current LFG. The experimental and control group baseline SPPB scores were 8.7 points and 8.9 points, respectively. The control group received written instructions on the intervention exercises and performed them independently, and the experimental group received in-clinic training and feedback on the exercises. After the intervention period, SPPB scores improved 1.4 points in the experimental group and did not change in the control group. These results support our hypothesis that a combined kyphosis exercise and posture training program in a low functioning cohort may improve physical function, but a larger study powered to detect a significant change in physical function is needed.

It is possible targeting kyphosis in older adults with low physical function and hyperkyphosis may slow expected age-related decline in physical function. The minimum clinically important difference (MCID) has been reported to be between 0.3 to 0.8 points on the SPPB using a combination of both distribution and anchor-based methods in two different studies evaluating cohorts similar to the current cohort (age range 7089 ) of mildly frail older adults. This suggests the 0.62 (95\%CI: -0.2 to 1.44 ) point improvement on the SPPB in the LFG may be clinically relevant $[45,46]$. Furthermore older adults in LFG improved from a baseline SPPB of 9.62 (1.2) to a score greater than the cut-off score of 10 suggesting targeting kyphosis may mitigate risk of future disability [22]. The LFG improved gaitspeed 0.04 (95\%CI: -0.02 to 0.11$) \mathrm{m} / \mathrm{s}$ which may be clinically relevant given MCID has been reported to be 
between 0.03 to $0.06 \mathrm{~m} / \mathrm{s}$ utilizing both distribution and anchor-based methods $[45,46]$. In fact, on average 3 years after the SCOR intervention, kyphosis was maintained and gait speed improved an additional $0.08 \mathrm{~m} / \mathrm{s}$, highlighting the potential long-term benefits of a shortterm kyphosis intervention [47]. Moreover, Merchant et al. [48] concluded trunk adaptations including flexed posture precede declines in gait speed in a crosssectional gait kinematics study of older Chinese men who were mildly frail when compared to fit older men. Our LFG may have been transitioning to frailty and improving kyphosis may have associated with a small improvement in gait speed, possibly slowing the progression to frailty.

We investigated change in self-image, which is central to psychological well-being and a measure of HRQoL and has been linked to kyphosis [1, 49]. Self-image, a subdomain of self-esteem, is related to exercise selfefficacy, one's belief in one's ability participate in regular exercise [50]. We observed a small within group improvement in self-image for both the LFG 0.19 (95\%CI: 0.02 to 0.37 ) points and the HFG 0.24 (95\%CI: 0.14 to 0.34 ) points after receiving the intervention and both groups responded simarly, $p=0.473$. These changes are less than the 0.43 (95\% CI: 0.24 to 0.61 ) point change reported after a 6-month targeted kyphosis intervention delivered 3 times a week [15]. Improving self-image through a targeted kyphosis intervention may be useful in facilitating maintence of physical activity in older adults; however, neither LFG or HFG group improved physical activity significantly after the intervention and it is possible that a 3-month intervention was not adequate to facilitate a change. Sinaki et al. conducted a 2 year home-based progressive back strengthening exercise intervention randomized trial in post-menopausal women and found physical activity improved significantly more in the intervention group, $p=0.009$, at the 2 year mark suggesting a longer exposure to kyphosis targeted intervention may lead to improved physical activity [51].

Spinal extensor strength and endurance decreased in the LFG, but increased in the HFG after the intervention $(-6.0 \%$ vs $+6.8 \%, p=0.018)$ and $(-8.8 \mathrm{~s}$ vs $+5.2 \mathrm{~s}, p=$ $0.080)$, respectively. Weakness in the spinal extensors increases risk of kyphosis progression and more recently trunk muscle composition has been linked to increased risk of kyphosis progression and decline in physical function [52-55]. The LFG had lower baseline spinal endurance (112 s vs $138 \mathrm{~s}$ ) and higher baseline pain scores (3.3 vs 2.0 points) which may have affected their ability to improve during the intervention, and they may have benefited from more time to accommodate to the strengthening intervention provided in the 3 month intervention. There is a dose-response relationship to strength training in older adults and while 12-weeks leads to improved strength, longer durations up to 53 weeks leads to greater improvements in muscular strength [56]. While trunk muscle composition may be an important biologic factor in decline in physical function in older adults, it is unknown if improved trunk muscle composition has a mediating effect on improved physical function. Future trials targeting older adults with hyperkyphosis and low physical function should consider assessing trunk muscle composition in future trials to investigate it as a mediating factor for change in physical function.

Several factors indicate the intervention was welltolerated in both the low- and high-functioning groups. Adherence to the intervention was similar in both groups. The LFG attended an average of 1 more class than the HFG participants (20 versus 19 of the possible 24 classes) and a similar number of people had nonreportable complaints in the LFG and HFG (69\% vs $68 \%)$. The LFG started the intervention with higher resting pain scores (3.3 vs 2.0 points); however, both groups had small non-significant reductions in pain over the 3 month intervention period ( -0.3 points in HFG, -0.2 points in LFG). There were no reportable adverse events in either group. The adherence rates, non-reportable events and reportable adverse events are consistent with findings reported in a meta analysis ( $n=13$ studies) evaluating the impact of exercise on hyperkyphotic posture in post-menopausal women and men [57]. These results suggest testing this intervention in a larger cohort of older adults who have low physical function is feasible and safe. Specific drop-out data within the HFG and LFG is unavailable which could have artificially influenced adherence and safety in either group. However, 9 participants dropped out in the first week due to lack of interest or time and 2 did not have analyzable baseline radiographs which reflects favorably on the integrity of the cohorts.

This analysis suggests that lower functioning older adults who are transitioning to frailty with hyperkyphosis may improve physical function after a targeted kyphosis intervention; however, there were several limitations. This was a post-hoc exploratory analysis of previously reported data from a randomized controlled trial. Causal relationships cannot be established because the number of older adults in the low functioning group was small, and the analysis was not powered to detect significant changes in physical function. Furthermore, we did not adjust for covariates due to the small sample size in our analyses, although we did perform age and sex-adjusted sensitivity analyses, and it is possible the changes in physical function were attributable to other covariates. There were differences in baseline characteristics of BMI, self-reported health, physical function and 
HrQol in the LFG and HFG that may have influenced results. The results in our LFG are not generalizable to a frail cohort of older adults given participants needed to be able to rise from a chair without their hands and walk a block without an assistive device to meet recruitment criteria. However we can generalize to a cohort of older adults who are transitioning to physical frailty based on a SPPB score of 10 or less. The difference in baseline SPPB scores were small (9.6 vs 11.4) which could limit generalizability to all older adults; however, there was a significant difference between change in SPPB score after controlling for age, thus this intervention may be generalizable to older adults transitioning to frailty regardless of age. Furthermore, for each unit increase in SPPB score, the odds of incident disability risk decrease by approximately $25 \%$, suggesting the small magnitude of difference between the LFG and HFG baseline SPPB scores is clinically relevant [20]. Despite the limitations, the magnitude and direction of changes in SPPB and gait speed suggest further study is warranted to test the hypothesis that lower functioning older adults who are transitioning to frailty with hyperkyphosis who participate in a targeted kyphosis intervention will improve physical function. The strength of this study is that no prior published studies have specifically targeted lower functioning older adults with hyperkyphosis to determine the effects of a kyphosis intervention on physical function. However, our results are consistent with other studies that included a range of physical functioning, including lower functioning older adults with hyperkyphosis $[17,18]$.

\section{Conclusion}

The results from this secondary analysis suggest that older adults with low physical function who are transitioning to frailty and hyperkyphosis may improve physical function after a targeted kyphosis intervention. Older adults with low physical function in our cohort adhered to and safely participated in a progressive, targeted high-intensity kyphosis exercise and posture training intervention. This observation needs to be confirmed in larger adequately powered studies. Further study is warranted with a large sample of low functioning older adults and hyperkyphosis to confirm these observations and determine if hyperkyphosis is relevant on the causal path towards decline in physical function and frailty.

\section{Supplementary Information}

The online version contains supplementary material available at https://doi. org/10.1186/s12877-021-02062-8.

Additional file 1:. Age-adjusted mean change pre/post intervention in outcome measures in the HFG, LFG with confidence intervals (Cl).
Additional file 2:. Age and sex adjusted mean change pre/post intervention in outcome measures in the HFG, LFG with confidence intervals $(\mathrm{Cl})$.

Additional file 3:. Numeric Results for Comparing Mean Change in a Repeated Measures Design - Sample size estimation to determine change in Short Physical Performance Battery (SPPB) in a low functioning cohort after a kyphosis intervention.

\section{Abbreviations \\ SCOR: Specialized Center of Research Kyphosis randomized trial; HrQol: Health-related Quality of Life; modified PPT: Modified Physical Performance Test; SPPB: Short Physical Performance Battery; LFG: Low function group; HFG: High function group; MCID: Minimum Clinically Important Difference; TUG: Timed Up and Go; 6 MW: Six Minute Walk test; PROMIS: Patient-Reported Outcome Measuremement Information System; PASE: Physical Activity Scale for the Elderly}

\section{Authors' contributions}

AG and WK participated in conception, design, analysis, interpretation and writing the manuscript, YF, NL participated in conception, design, analysis, interpretation and reviewing the manuscript, NP and SW participated in acquisition, analysis and interpretation of the work and reviewing the manuscript. The author(s) read and approved the final manuscript.

\section{Funding}

The original SCOR trial was supported by grant P50 AR063043 from the Office of Research in Women's Health and the National Institute of Arthritis and Musculoskeletal and Skin Disorders (PI: Lane). This work was supported by grant K24NR015812 from the National Institute of Nursing Research (PI: Fukuoka).

\section{Availability of data and materials}

The datasets used and/or analysed during the current study are available from the corresponding author on reasonable request.

\section{Ethics approval and consent to participate}

This study was approved by the Institutional Review Boards for the University of California at San Francisco and Kaiser Permanente Northern California. Written informed consent was obtained from all participants. The study protocol and methods were performed in accordance with the guidelines and regulations of the Declaration of Helsinki.

Consent for publication

Not applicable.

\section{Competing interests}

No authors report competing interests.

\section{Author details}

${ }^{1}$ Chronic Pain Management, San Francisco Kaiser Permanente Medical Center, 4141 Geary Blvd, Suite 212, San Francisco, CA 94118, USA.

${ }^{2}$ Department of Physical Therapy and Rehabilitation Science, University of California, San Francisco, 1500 Owens Street, Suite 400, San Francisco, CA 94143 , USA. ${ }^{3}$ Department of Physiological Nursing, School of Nursing, University of California, San Francisco, 2 Koret Way, San Francisco, CA 94143, USA. ${ }^{4}$ California Pacific Medical Center, Research Institute, San Francisco, CA, USA. ${ }^{5}$ Center for Musculoskeletal Health, University of California at Davis School of Medicine, 4625 2nd Ave, Suite 200, Davis, CA 95817, USA.

Received: 19 November 2020 Accepted: 29 January 2021

Published online: 22 February 2021

\section{References}

1. Roghani T, Zavieh MK, Manshadi FD, King N, Katzman W. Age-related hyperkyphosis: update of its potential causes and clinical impacts-narrative review. Aging Clin Exp Res. 2017;29(4):567-77.

2. Katzman WB, Vittinghoff E, Kado DM. Age-related hyperkyphosis, independent of spinal osteoporosis, is associated with impaired mobility in older community-dwelling women. Osteoporos Int. 2011;22(1):85-90. 
3. Katzman WB, Vittinghoff E, Ensrud K, Black DM, Kado DM. Increasing kyphosis predicts worsening mobility in older community-dwelling women: a prospective cohort study. J Am Geriatr Soc. 2011;59(1):96-100.

4. Katzman WB, Huang MH, Lane NE, Ensrud KE, Kado DM. Kyphosis and decline in physical function over 15 years in older community-dwelling women: the study of osteoporotic fractures. J Gerontol A Biol Sci Med Sci. 2013;68(8):976-83.

5. Eum R, Leveille SG, Kiely DK, Kiel DP, Samelson EJ, Bean JF. Is kyphosis related to mobility, balance, and disability? Am J Phys Med Rehabil. 2013; 92(11):980-9.

6. Antonelli-Incalzi R, Pedone C, Cesari M, Di lorio A, Bandinelli S, Ferrucci L. Relationship between the occiput-wall distance and physical performance in the elderly: a cross sectional study. Aging Clin Exp Res. 2007;19(3):207-12.

7. Kado DM, Lui LY, Ensrud KE, Fink HA, Karlamangla AS, Cummings SR. Hyperkyphosis predicts mortality independent of vertebral osteoporosis in older women. Ann Intern Med. 2009;150(10):681-7.

8. Huang MH, Barrett-Connor E, Greendale GA, Kado DM. Hyperkyphotic posture and risk of future osteoporotic fractures: the rancho Bernardo study J Bone Miner Res. 2006;21(3):419-23.

9. McDaniels-Davidson C, Davis A, Wing D, et al. Kyphosis and incident falls among community-dwelling older adults. Osteoporos Int. 2018;29(1):163-9.

10. van der Jagt-Willems HC, de Groot MH, van Campen JP, Lamoth CJ, Lems WF. Associations between vertebral fractures, increased thoracic kyphosis, a flexed posture and falls in older adults: a prospective cohort study. BMC Geriatr. 2015;15:34

11. Ziebart C, Gibbs JC, MCArthur C, et al. Are osteoporotic vertebral fractures or forward head posture associated with performance-based measures of balance and mobility? Arch Osteoporos. 2019;14(1):67.

12. Kado DM, Miller-Martinez D, Lui LY, et al. Hyperkyphosis, kyphosis progression, and risk of non-spine fractures in older community dwelling women: the study of osteoporotic fractures (SOF). J Bone Miner Res. 2014; 29(10):2210-6.

13. Lorbergs AL, Murabito JM, Jarraya M, et al. Thoracic kyphosis and physical function: the Framingham study. J Am Geriatr Soc. 2017;65(10):2257-64.

14. Katzman WB, Parimi N, Gladin A, et al. Sex differences in response to targeted kyphosis specific exercise and posture training in communitydwelling older adults: a randomized controlled trial. BMC Musculoskelet Disord. 2017;18(1):509.

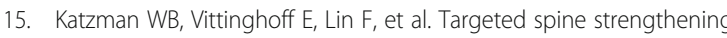
exercise and posture training program to reduce hyperkyphosis in older adults: results from the study of hyperkyphosis, exercise, and function (SHEA F) randomized controlled trial. Osteoporos Int. 2017;28(10):2831-41.

16. Greendale GA, Huang MH, Karlamangla AS, Seeger L, Crawford S. Yoga decreases kyphosis in senior women and men with adult-onset hyperkyphosis: results of a randomized controlled trial. J Am Geriatr Soc. 2009;57(9):1569-79.

17. Benedetti MG, Berti L, Presti C, Frizziero A, Giannini S. Effects of an adapted physical activity program in a group of elderly subjects with flexed posture: clinical and instrumental assessment. J Neuroeng Rehabil. 2008;5:32.

18. Jang HJ, Hughes LC, Oh DW, Kim SY. Effects of corrective exercise for thoracic Hyperkyphosis on posture, balance, and well-being in older women: a doubleblind, Group-Matched Design. J Geriatr Phys Ther. 2019;42(3):E17-27.

19. Guralnik JM, Ferrucci L, Simonsick EM, Salive ME, Wallace RB. Lowerextremity function in persons over the age of 70 years as a predictor of subsequent disability. N Engl J Med. 1995;332(9):556-61.

20. Vasunilashorn S, Coppin AK, Patel KV, et al. Use of the short physical performance battery score to predict loss of ability to walk 400 meters: analysis from the InCHIANTI study. J Gerontol A Biol Sci Med Sci. 2009;64(2):223-9.

21. Minneci C, Mello AM, Mossello E, et al. Comparative study of four physical performance measures as predictors of death, incident disability, and falls in unselected older persons: the insufficienza Cardiaca negli Anziani Residenti a Dicomano study. J Am Geriatr Soc. 2015;63(1):136-41.

22. Perracini MR, Mello M, de Oliveira Máximo R, et al. Diagnostic Accuracy of the Short Physical Performance Battery for Detecting Frailty in Older People. Phys Ther. 2020;100(1):90-98.

23. Pavasini R, Guralnik J, Brown JC, et al. Short physical performance battery and all-cause mortality: systematic review and meta-analysis. BMC Med. 2016;14(1):215

24. Freiberger $E$, de Vreede $P$, Schoene $D$, et al. Performance-based physical function in older community-dwelling persons: a systematic review of instruments. Age Ageing. 2012;41(6):712-21.
25. Brodaty H, Low LF, Gibson L, Burns K. What is the best dementia screening instrument for general practitioners to use? Am J Geriatr Psychiatry. 2006; 14(5):391-400

26. Katzman WB, Vittinghoff E, Kado DM, et al. Study of Hyperkyphosis, exercise and function (SHEAF) protocol of a randomized controlled trial of multimodal spine-strengthening exercise in older adults with Hyperkyphosis. Phys Ther. 2016;96(3):371-81.

27. Lundon KM, Li AM, Bibershtein S. Interrater and intrarater reliability in the measurement of kyphosis in postmenopausal women with osteoporosis. Spine (Phila Pa 1976). 1998;23(18):1978-85.

28. Grados F, Fechtenbaum J, Flipon E, Kolta S, Roux C, Fardellone P. Radiographic methods for evaluating osteoporotic vertebral fractures. Joint Bone Spine. 2009;76(3):241-7.

29. Genant HK, Wu CY, van Kuijk C, Nevitt MC. Vertebral fracture assessment using a semiquantitative technique. J Bone Miner Res. 1993;8(9):1137-48.

30. Resnick D, Shapiro RF, Wiesner KB, Niwayama G, Utsinger PD, Shaul SR. Diffuse idiopathic skeletal hyperostosis (DISH) [ankylosing hyperostosis of Forestier and rotes-Querol]. Semin Arthritis Rheum. 1978;7(3):153-87.

31. Bennell K, Dobson F, Hinman R. Measures of physical performance assessments: self-paced walk test (SPWT), stair climb test (SCT), six-minute walk test (6MWT), chair stand test (CST), timed up \& go (TUG), sock test, lift and carry test (LCT), and Car task. Arthritis Care Res. 2011;63(Suppl 11):S350 70.

32. Steffen TM, Hacker TA, Mollinger L. Age- and gender-related test performance in community-dwelling elderly people: six-minute walk test, berg balance scale, timed up \& go test, and gait speeds. Phys Ther. 2002; 82(2):128-37.

33. Binder EF, Storandt M, Birge SJ. The relation between psychometric test performance and physical performance in older adults. J Gerontol A Biol Sci Med Sci. 1999:54(8):M428-32.

34. Brown M, Sinacore DR, Binder EF, Kohrt WM. Physical and performance measures for the identification of mild to moderate frailty. J Gerontol A Biol Sci Med Sci. 2000;55(6):M350-5

35. Gershon RC, Rothrock N, Hanrahan R, Bass M, Cella D. The use of PROMIS and assessment center to deliver patient-reported outcome measures in clinical research. J Appl Meas. 2010;11(3):304-14.

36. Hays RD, Bjorner JB, Revicki DA, Spritzer KL, Cella D. Development of physical and mental health summary scores from the patient-reported outcomes measurement information system (PROMIS) global items. Qual Life Res. 2009;18(7):873-80.

37. Asher MA, Min Lai S, Burton DC. Further development and validation of the Scoliosis Research Society (SRS) outcomes instrument. Spine (Phila Pa 1976). 2000;25(18):2381-6.

38. Washburn RA, Smith KW, Jette AM, Janney CA. The physical activity scale for the elderly (PASE): development and evaluation. J Clin Epidemiol. 1993; 46(2):153-62.

39. Hays RD, Spritzer KL, Thompson WW, Cella D. U.S. general population estimate for "excellent" to "poor" self-rated health item. J Gen Intern Med. 2015;30(10):1511-6.

40. Katzman WB, Sellmeyer DE, Stewart AL, Wanek L, Hamel KA. Changes in flexed posture, musculoskeletal impairments, and physical performance after group exercise in community-dwelling older women. Arch Phys Med Rehabil. 2007;88(2):192-9.

41. Shipp KM, Purse JL, Gold DT, et al. Timed loaded standing: a measure of combined trunk and arm endurance suitable for people with vertebral osteoporosis. Osteoporos Int. 2000;11(11):914-22.

42. Greendale GA, Nili NS, Huang MH, Seeger L, Karlamangla AS. The reliability and validity of three non-radiological measures of thoracic kyphosis and their relations to the standing radiological cobb angle. Osteoporos Int. 2011; 22(6):1897-905.

43. Ohlen $G$, Spangfort $E$, Tingvall $C$. Measurement of spinal sagittal configuration and mobility with Debrunner's kyphometer. Spine (Phila Pa 1976). 1989;14(6):580-3.

44. Armstrong RB. Mechanisms of exercise-induced delayed onset muscular soreness: a brief review. Med Sci Sports Exerc. 1984;16(6):529-38.

45. Perera S, Mody SH, Woodman RC, Studenski SA. Meaningful change and responsiveness in common physical performance measures in older adults. J Am Geriatr Soc. 2006;54(5):743-9.

46. Kwon S, Perera S, Pahor $M$, et al. What is a meaningful change in physical performance? Findings from a clinical trial in older adults (the LIFE-P study). J Nutr Health Aging. 2009;13(6):538-44. 
47. Katzman WB, Parimi N, Gladin A, Wong S, Lane NE. Long-term efficacy of treatment effects after a kyphosis exercise and posture training intervention in older community-dwelling adults: a cohort study. J Geriatr Phys Ther. 2020.

48. Merchant RA, Banerji S, Singh G, et al. Is trunk posture in walking a better marker than gait speed in predicting decline in function and subsequent frailty? J Am Med Dir Assoc. 2016;17(1):65-70.

49. McAuley E, Elavsky S, Motl RW, Konopack JF, Hu L, Marquez DX. Physical activity, self-efficacy, and self-esteem: longitudinal relationships in older adults. J Gerontol B Psychol Sci Soc Sci. 2005;60(5):268-75.

50. Elavsky S, McAuley E, Motl RW, et al. Physical activity enhances long-term quality of life in older adults: efficacy, esteem, and affective influences. Ann Behav Med. 2005;30(2):138-45.

51. Sinaki MIE, Wahner W, Wollan P. Stronger Back muscles reduce the incidence of vertebral fractures: a prospective 10 year follow-up of postmenopausal women. Bone. 2002;30(6):836-41.

52. Lorbergs AL, Allaire BT, Yang L, et al. A longitudinal study of trunk muscle properties and severity of thoracic kyphosis in women and men: the Framingham study. J Gerontol A Biol Sci Med Sci. 2019;74(3):420-7.

53. Hicks GE, Simonsick EM, Harris TB, et al. Trunk muscle composition as a predictor of reduced functional capacity in the health, aging and body composition study: the moderating role of back pain. J Gerontol A Biol Sci Med Sci. 2005;60(11):1420-4.

54. Sions JM, Coyle PC, Velasco TO, Elliott JM, Hicks GE. Multifidi muscle characteristics and physical function among older adults with and without chronic Low Back pain. Arch Phys Med Rehabil. 2017:98(1):51-7.

55. Katzman W, Cawthon P, Hicks GE, et al. Association of spinal muscle composition and prevalence of hyperkyphosis in healthy communitydwelling older men and women. J Gerontol A Biol Sci Med Sci. 2012;67(2): $191-5$.

56. Borde R, Hortobágyi T, Granacher U. Dose-response relationships of resistance training in healthy old adults: a systematic review and Metaanalysis. Sports Med. 2015;45(12):1693-720.

57. Bansal S, Katzman WB, Giangregorio LM. Exercise for improving age-related hyperkyphotic posture: a systematic review. Arch Phys Med Rehabil. 2014; 95(1):129-40.

\section{Publisher's Note}

Springer Nature remains neutral with regard to jurisdictional claims in published maps and institutional affiliations.

Ready to submit your research? Choose BMC and benefit from:

- fast, convenient online submission

- thorough peer review by experienced researchers in your field

- rapid publication on acceptance

- support for research data, including large and complex data types

- gold Open Access which fosters wider collaboration and increased citations

- maximum visibility for your research: over $100 \mathrm{M}$ website views per year

At $\mathrm{BMC}$, research is always in progress.

Learn more biomedcentral.com/submissions 